\title{
Stage IIB Penile Cancer AJCC v8
}

National Cancer Institute

\section{Source}

National Cancer Institute. Stage IIB Penile Cancer A/CC v8. NCI Thesaurus. Code

C140083.

Stage IIB includes: T3, N0, M0. T3: Penile cancer with tumor invading into corpora cavernosum (including tunica albuginea) with or without urethral invasion. N0: No lymph node metastasis. M0: No distant metastasis. (AJCC 8th ed.) 